\title{
STAR FORMATION HISTORIES OF BRIGHTEST CLUSTER GALAXIES WITH INTERMEDIATE CENTRAL AGES
}

\author{
J. Umanzor and M. L. Talavera \\ Universidad Nacional Autónoma de Honduras, Facultad de Ciencias Espaciales, Departamento de Astronomía \\ y Astrofísica.
}

Received February 16 2021; accepted July 142021

\begin{abstract}
This work is devoted to the study of the star formation histories (SFHs) of the brightest cluster galaxies (BCGs) with intermediate central ages (from 5 to $10 \mathrm{Gyr}$ ), to confirm if BCGs with these ages represent different accretion histories or simply a stochastic effect. The sample is composed of 6 BCGs with intermediate central ages and 3 BCGs with old central ages (>12 Gyr) as comparison galaxies. The galaxies were observed with the integrated field spectrograph VIMOS installed in the Very Large Telescope (VLT). The SFHs were obtained with the full spectrum fitting technique using the star population code STARLIGHT. The BCGs of intermediate central age analyzed formed almost $100 \%$ of their stars at $z>2$ and their SFHs are similar to the SFHs of BCGs of old central ages and elliptical galaxies of similar mass $\left(\mathrm{M}_{\mathrm{Dyn}}>10^{11} M_{\odot}\right)$; therefore, these BCGs do not represent different SFHs.
\end{abstract}

\section{RESUMEN}

Este trabajo está dedicado al estudio de las historias de formación estelar (HFE) de BCGs (brightest cluster galaxies) con edades centrales intermedias (de 5 a $10 \mathrm{Ga}$ ), para confirmar si BCGs con estas edades representan historias de acreción distintas o si es simplemente un efecto estocástico. La muestra está compuesta de 6 BCGs con edades intermedias y 3 BCGs con edades centrales viejas (> $12 \mathrm{Ga}$ ) como galaxias de comparación. Las galaxias se observaron con el espectrógrafo de campo integrado VIMOS instalado en el VLT (Very Large Telescope). Las HFE se obtuvieron con la técnica de ajuste de todo el espectro usando el código de poblaciones estelares STARLIGHT. Las BCGs de edades centrales intermedias analizadas formaron casi el $100 \%$ de sus estrellas a $z>2$ y sus HFE son similares a las HFE de BCGs de edades centrales viejas y de galaxias elípticas de masa similar $\left(\mathrm{M}_{\text {Din }}>10^{11} M_{\odot}\right)$; por tanto, estas BCGs no representan HFE distintas.

Key Words: galaxies: clusters: general - galaxies: elliptical and lenticular, $\mathrm{cD}$ galaxies: evolution - galaxies: formation - galaxies: star formation

\section{INTRODUCTION}

Brightest cluster galaxies (BCGs) are the largest and most luminous galaxies in the Universe, generally very close to the space and kinematic center of galaxy clusters. At first BCGs appear to be giant elliptical galaxies. However, they have unique properties (shallower surface brightness profiles, high speed scattering, relatively high luminosities and masses), and their special formation environment makes them different from giant elliptical galaxies. The following formation theories have been proposed to explain the origin of BCGs: cooling flows (Silk 1976; Cowie
\& Binney 1977; Fabian 1994), galactic cannibalism (Ostriker \& Tremaine 1975; White 1976; Malumuth \& Richstone 1984; Merritt 1985) and two-phase (De Lucia \& Blaizot 2007; Naab et al. 2009; Laporte et al. 2013). However, when these theories were tested in different cosmological settings, not all of them gave realistic results.

The first studies of BCGs focused on their high luminosities. BCGs are typically 10 times more luminous than normal elliptical galaxies (Sandage \& Hardy 1973; Schombert 1986), with absolute magnitudes between -21.5 to -23.5 in the $V$ band. Other 
studies found that the luminosities of BCGs are too high to be simply the brightest member of a standard luminosity function (Schechter \& Peebles 1976) of elliptical galaxies (e.g. Tremaine \& Richstone 1977; Dressler 1978; Bernstein \& Bhavsar 2001).

BCGs have usually been classified into two morphological types, $\mathrm{cD}$ galaxies that have faint and extended stellar halos and giant ellipticals, without an envelope. This is based on the morphology shown by BCGs in the optical range and their colors in the optical and near infrared, which suggest relatively old stellar populations and little ongoing star formation activity (Dubinski 1998; Tonini et al. 2012; Bai et al. 2014; Zhao et al. 2015). However, they distinguish themselves from the typical cluster elliptical galaxy by their relatively large masses $\left(\approx 10^{13} M_{\odot}\right.$, Katayama et al. (2003)) and luminosities, and by shallower surface brightness profiles and central velocity dispersions of $300-400 \mathrm{~km} \mathrm{~s}^{-1}$ (Oemler 1976; Schombert 1986; Dubinski 1998; Edwards et al. 2020). Von der Linden et al. (2007) found BCGs to have a higher dynamical-to-stellar mass ratio, indicating that they contained a larger fraction of dark matter compared to a sample of nonBCG galaxies. Also, the BCGs often show double or multiple nuclei (Schneider et al. 1983; Laine et al. 2003).

By using integral field unit (IFU) spectroscopy Oliva-Altamirano et al. (2015) performed an analysis of the stellar populations of 9 BCGs at $z<0.1$. They showed that BCGs have high central metallicity, flat metallicity gradients and ages in a wide range of 5 to 15 Gyr. They also found that $67 \%(6 / 9)$ of BCGs have intermediate central ages $(5 \mathrm{Gyr}<$ age $<10 \mathrm{Gyr}$ ) suggesting a history of active accretion (star formation up to $z \approx 1$ ) and $33 \%(3 / 9)$ have old central ages (age $>11 \mathrm{Gyr}$ ) reflecting that they have not had star formation since $z \approx 2$. The stellar population measurements come from the luminosityweighed distribution and are sensitive to the brightest and youngest populations of the stars in the galaxy (e.g. Trager \& Somerville 2009).

Hydrodynamic simulations of early type galaxies (ETGs) in environments less dense than those typically found around BCGs predict that more massive galaxies are older than less massive galaxies, such that at masses $>10^{10.5} M_{\odot}$ the galaxies are older than 10 Gyr (Naab et al. 2014; Peeples et al. 2014; Hirschmann et al. 2013) and show passive evolution from $z \approx 2$.

The SFH is a function that describes how the rate of star formation varies over cosmic time until the moment of observation. The $\mathrm{SFH}$ in galaxies is one of the most important parameters to constrain cosmological models, the formation and evolution of galaxies and the star formation theories. Knowledge of SFH is important for the development of various topics in the evolution of galaxies, such as chemical enrichment, interactions between galaxies as dissipators of star formation, and the role played by stellar dynamics and the self-propagation of star formation. In particular, the SFH of BCGs provide information about their formation conditions in the early universe and also about the mechanism by which star formation shuts down.

In this work, the SFH of BCGs of intermediate and old central ages are inferred using the sample of BCGs from Oliva-Altamirano et al. (2015). The SFH were obtained with the whole spectrum fitting technique using the stellar population code STARLIGHT (Cid Fernandes et al. 2005). We investigated whether BCGs with intermediate central ages have different accretion histories, or whether it is simply a stochastic effect. The outline of this paper is as follows. In $\S 2$ we describe observations, data reduction and spectrum fitting. We present the results in $\S 3$. These are later discussed in $\S 4$. For the analysis of the results we adopt the $\Lambda$ CDM cosmology with $\Omega_{M}=0.27$ and $H_{o}=70 \mathrm{Km} \mathrm{s}^{-1} \mathrm{Mpc}^{-1}$.

\section{METHODOLOGY}

\subsection{Observations}

The observations of the BCGs were carried out using an IFU. An IFU is an instrument that divides the field of view into many spaxels (cells or spatial pixels). In astronomy, IFUs are used to study large objects such as nebulae, galaxies or a crowded cluster of stars or galaxies in one shot, using a technique known as integral field spectroscopy. In this method, the signal from each spaxel in the field of view is fed into a spectrograph, which generates a spectrum for each individual spaxel. With all the resulting spectra, a data cube is formed, which contains everything in the 2D field of view, in addition to the third dimension obtained by the spectrograph.

The sample is composed of 6 BCGs with intermediate central ages and 3 BCGs with old central ages (See Table 1). The ages were calculated by a stellar population analysis (Olivia-Altamirano et al. 2015). The BCGs are part of the C4 Cluster Cata$\log$ (Miller et al. 2005) from the Third Data Release (DR3) from the Sloan Digital Sky Survey (SDSS; York et al. 2000). BCGs were observed with the VLT using the VIMOS spectrograph (Le Fèvre et al. 
TABLE 1

BCGS OF INTERMEDIATE AND OLD CENTRAL AGES

\begin{tabular}{|c|c|c|c|c|c|}
\hline $\begin{array}{l}\mathrm{BCGs}^{\text {a }} \\
\text { (SDSS-C4-DR3) }\end{array}$ & $\begin{array}{c}\mathrm{R}_{e}{ }^{\mathrm{b}} \\
{\left[{ }^{\prime \prime}\right]}\end{array}$ & $z^{\mathrm{b}}$ & $\begin{array}{c}\log \mathrm{M}_{D y n} \mathrm{~b}, \mathrm{c} \\
{\left[M_{\odot}\right]}\end{array}$ & $\begin{array}{l}\text { Age }^{\mathrm{d}} \\
{[\mathrm{Gyr}]}\end{array}$ & Merging? d \\
\hline \multicolumn{6}{|c|}{ Intermediate age } \\
\hline 1027 & 6.98 & 0.090 & 11.79 & 8.9 & $\mathrm{y}$ \\
\hline 1042 & 7.22 & 0.094 & 11.83 & 6.4 & $\mathrm{n}$ \\
\hline 1048 & 5.17 & 0.077 & 11.59 & 7.1 & $\mathrm{y}$ \\
\hline 1066 & 4.95 & 0.083 & 11.62 & 5.8 & $\mathrm{y}$ \\
\hline 1261 & 5.76 & 0.037 & 11.32 & 8.8 & $\mathrm{n}$ \\
\hline 2039 & 8.82 & 0.082 & 11.86 & 9.6 & $\mathrm{n}$ \\
\hline \multicolumn{6}{|c|}{ Old age } \\
\hline 1050 & 8.43 & 0.072 & 11.78 & 16.4 & $\mathrm{n}$ \\
\hline 2001 & 5.84 & 0.041 & 11.38 & 12.6 & $\mathrm{n}$ \\
\hline 2086 & 4.83 & 0.083 & 11.60 & 13.2 & $\mathrm{y}$ \\
\hline
\end{tabular}

${ }^{a}$ We use the same nomenclature as Jimmy et al. (2013) and Oliva-Altamirano et al. (2015), i.e. we present each cluster by the last four digits of the SDSS flag, rather than the SSDS-C4-DR3 number.

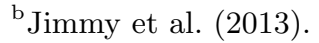

${ }^{\mathrm{c}}$ The dynamical mass was calculated using the standard equation given in Cappellari et al. (2006).

${ }^{\mathrm{d}}$ Oliva-Altamirano et al. (2015).

2003). VIMOS was used in IFU mode with the highresolution blue grism, which has a spectral resolution of $0.51 \AA$ pixel $^{-1}$. The galaxies observed with the instruments mentioned above have a spatial sampling of $0^{\prime \prime} 67 \mathrm{pixel}^{-1}$ and a field of view of $27^{\prime \prime} \times 27^{\prime \prime}$. The observations were made in two parts: from April to August 2008 (Prog. ID 381.B-0728) and from April to July 2011 (ID 087.B-0366), during dark nights with an average seeing of 0.9 .

\subsection{Data Reduction}

The reduction of the IFU data was done first using the VIMOS code (Izzo et al. 2004). This code generates the calibration files (master bias, arc spectrum, flat field, fiber identification, etc.) and extracts the science spectrum in every fiber of every image. The VIMOS field of view is made up of four quadrants and the VIMOS code reduces each quadrant separately. Second, an IDL routine is used to mask the bad fibers, subtract the sky in each of the quadrants of the science images, and then combine the quadrants to form a data cube. More details on the observations and data reduction can be found in Jimmy et al. (2013) and Oliva-Altamirano et al. (2015).

\subsection{Spectrum Fitting}

After generating the data cube for each galaxy we used the PYTHON code of Oliva-Altamirano et al. (2015) to obtain the spectrum of the center of each BCG. This code divides each galaxy into rings and then the spaxels (spectrum of a region of the field of view) of each ring are stacked to obtain one spectrum per ring. The code identifies the rings following the total flow, therefore the shape of the rings is not circular but rather defined by the morphology of the galaxy. The spectrum of each spaxel of each galaxy was run at the wavelength of the rest frame before being stacked using the speed calculated with the program pPXF (Penalized Pixel Fitting) of Emsellem et al. (2007). pPXF fits stellar pattern libraries to the characteristic absorption lines of the BCG spectra, giving the recession rates of the spectral lines.

The left panel of Figure 1 shows the annular distribution of BCG 2039. The right panel is the fluxcollapsed VIMOS image. The annuli are 0.2, 0.48, 0.77 and $1.0 \mathrm{R}_{e}$ in each galaxy. The central aperture has been defined as $0.2 \pm 0.03 \mathrm{R}_{e}$. In Table 1 , we show $\mathrm{R}_{e}$ for each galaxy. BCG 1027 has $z=0.09$ and $\mathrm{R}_{e}=66^{\prime \prime} 98$; for this galaxy $\mathrm{R}_{e}$ corresponds to $\approx 13 \mathrm{kpc}$. 


\section{\begin{tabular}{|l|l|}
\hline & $0.2 \mathrm{Re}$ \\
$\square$ & $0.48 \mathrm{Re}$ \\
$0.77 \mathrm{Re}$ \\
$\square \quad$ \\
$1.0 \mathrm{Re}$ \\
\hline$\square$
\end{tabular}}

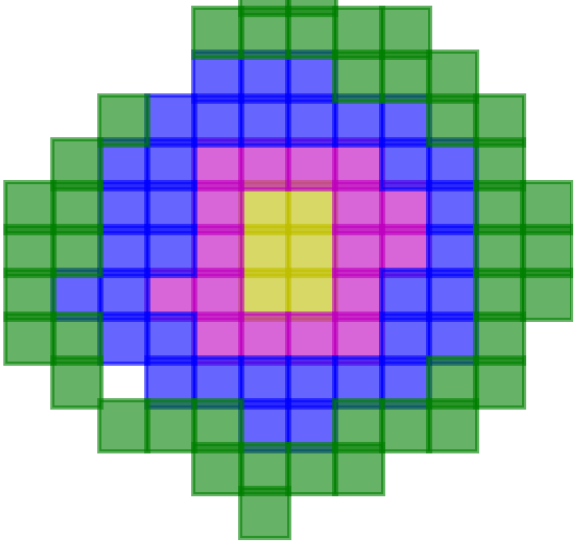

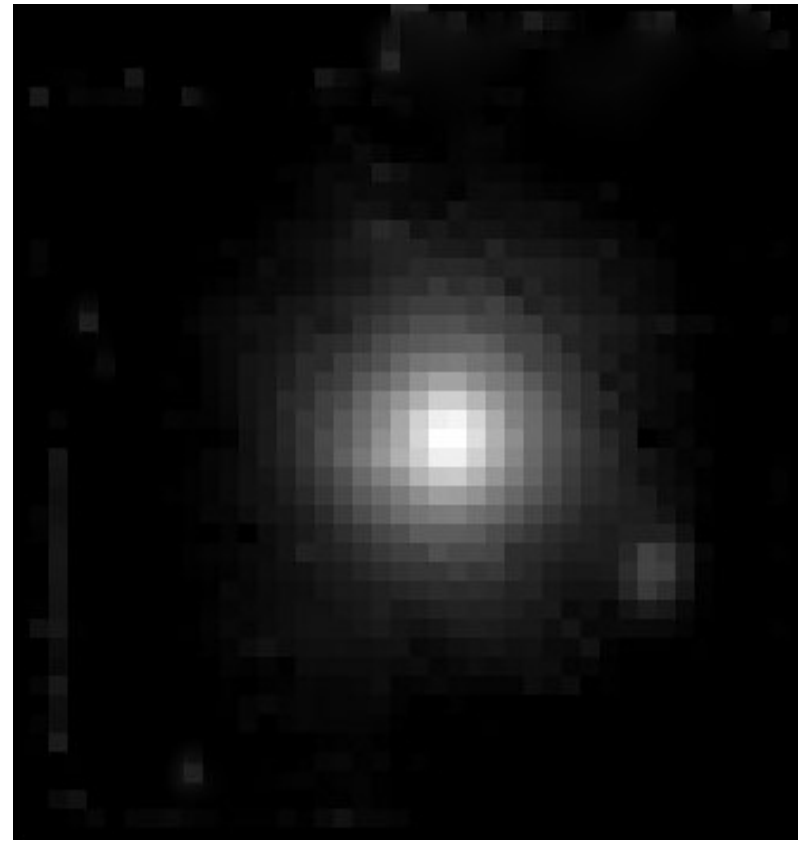

Fig. 1. Left Panel: The annular distribution of BCG 2039. The central annulus, corresponds to the aperture $0.2 \pm 0.03 \mathrm{R}_{e}$. The annuli all extend to $1 \mathrm{R}_{e}$. Right Panel: The flux-collapsed VIMOS image BCG 2039. The color figure can be viewed online.

Finally, with the STARLIGHT code, the SFHs of the BCGs were determined. STARLIGHT is a synthesis code for stellar populations. The method is based on fitting the observed spectrum of the galaxy as a linear combination of spectra of simple stellar populations. The adjustment is carried out through the techniques of simulated annealing, Metropolis and Markov Chain Monte Carlo. STARLIGHT fits an observed spectrum with a combination of $\mathrm{N}_{\star}$ simple stellar populations from the evolutionary synthesis models of Bruzual and Charlot (2003). In this work we use a base that contains $\mathrm{N}_{\star}=45$ spectra of 3 different metallicities $\left(Z=0.2,1.0,2.5 Z_{\odot}\right)$ and 15 ages between $1 \mathrm{Myr}$ and $13 \mathrm{Gyr}$. The stellar extinction is modeled by the extinction law of Cardelli et al. (1989) with $R V=3.1$.

Figures 2 and 3 illustrate the spectrum fits obtained for BCGs. The upper left panel shows the observed spectrum (black line) and the model (red line). The lower left panel shows the residual spectrum (observed - model). The histograms in the right panel show the SFHs as \% of the light fraction at $4020 \AA$ (upper) and as \% of the mass fraction (lower) both decomposed into 15 ages and 3 metallicities. Some of the derived properties are shown in the upper right: $\chi_{\lambda}^{2}$ is the fit $\chi^{2}$ divided by the number of $\lambda$ 's used in the fit; adev is the mean relative dif- ference between the model and observed spectrum; $\mathrm{S} / \mathrm{N}$ is the signal to noise ratio in the region centered at $\lambda=5000 \AA$.

\section{RESULTS}

In this section we use the integrated spectrum fit with an aperture of $0.2 \pm 0.03 \mathrm{R}_{e}$ to investigate the SFHs of 6 BCGs (SDSS-C4-DR3 1027, SDSS-C4DR3 1042, SDSS-C4-DR3 1048, SDSS-C4-DR3 1066, SDSS-C4-DR3 1261 and SDSS-C4-DR3 2039) of intermediate central ages. In addition, the fits of 3 BCGs (SDSS-C4-DR3 1050, SDSS-C4-DR3 2001 and SDSS-C4-DR3 2086) of old central ages are inferred.

Since a composite stellar population can be seen as the sum of simple stellar populations (Bruzual \& Charlot 2003), the SFH of a stellar population can be understood as the fraction of stellar mass produced as a function of time in the form of simple stellar populations. Therefore, the mass fraction $\mu_{j}$ provided by STARLIGHT plotted as function of the simple stellar populations ages can be considered as direct proxy for the output SFH (Citro et al. 2016).

In Figure 4 the SFHs of BCGs of intermediate central ages are shown. The SFHs of these galaxies are formed with few stellar populations of different ages (less than 4). 83\% (5/6) of the BCGs $(1027,1048,1066,1261$ and 2039) of intermediate 

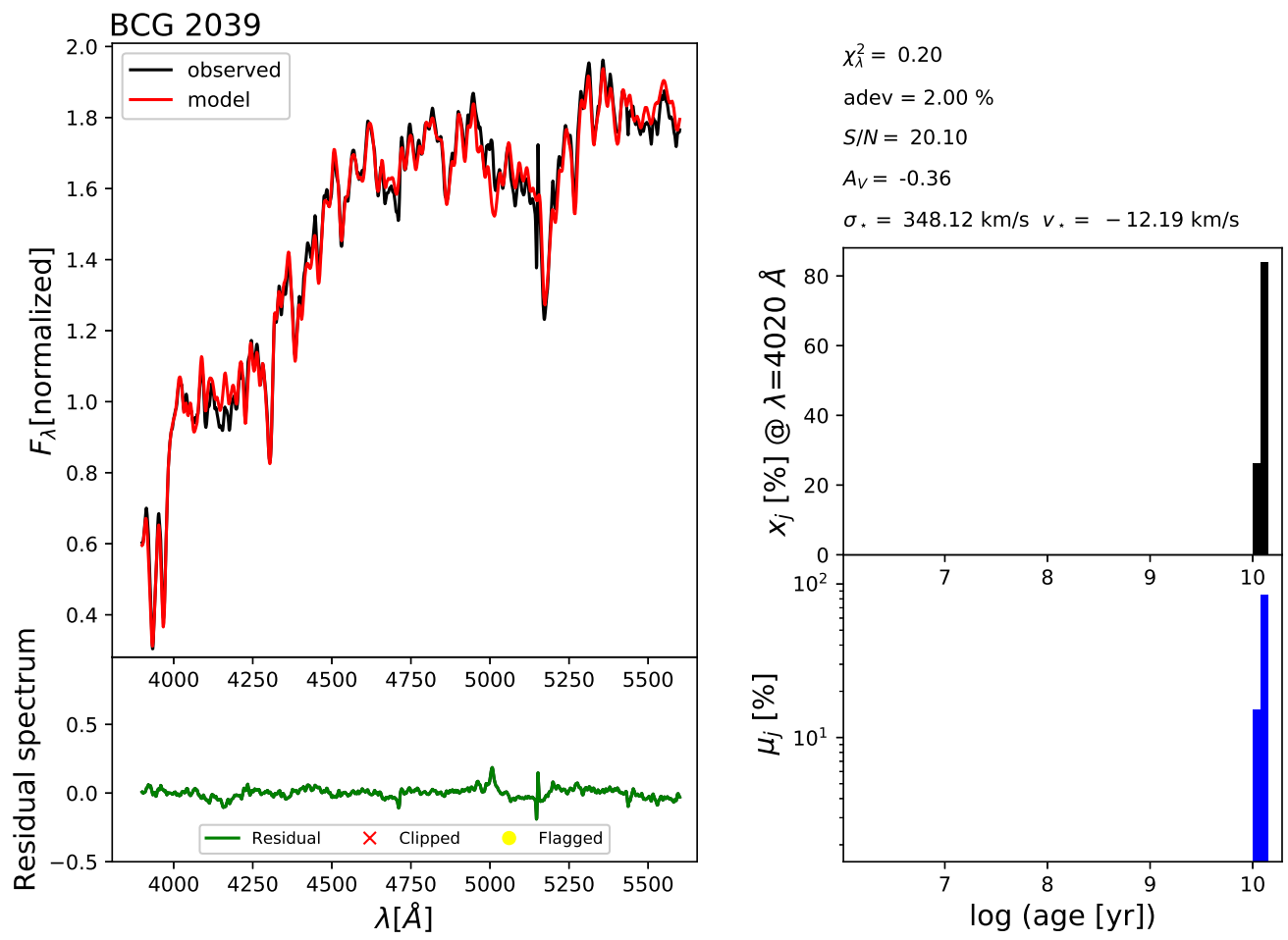

Fig. 2. Spectral synthesis of an intermediate age BCG. Top left: Observed (black line) and model (red line). Bottom left: residual spectrum (green line). Right: Flux (top) and mass (bottom) fractions as a function of age. Some of the derived properties are listed in the top right: $\chi_{\lambda}^{2}$ is the reduced $\chi^{2}$, adev is is the mean relative difference between model and observed spectra and $\mathrm{S} / \mathrm{N}$ refers to the region around $\lambda=5000 \AA$. The color figure can be viewed online.
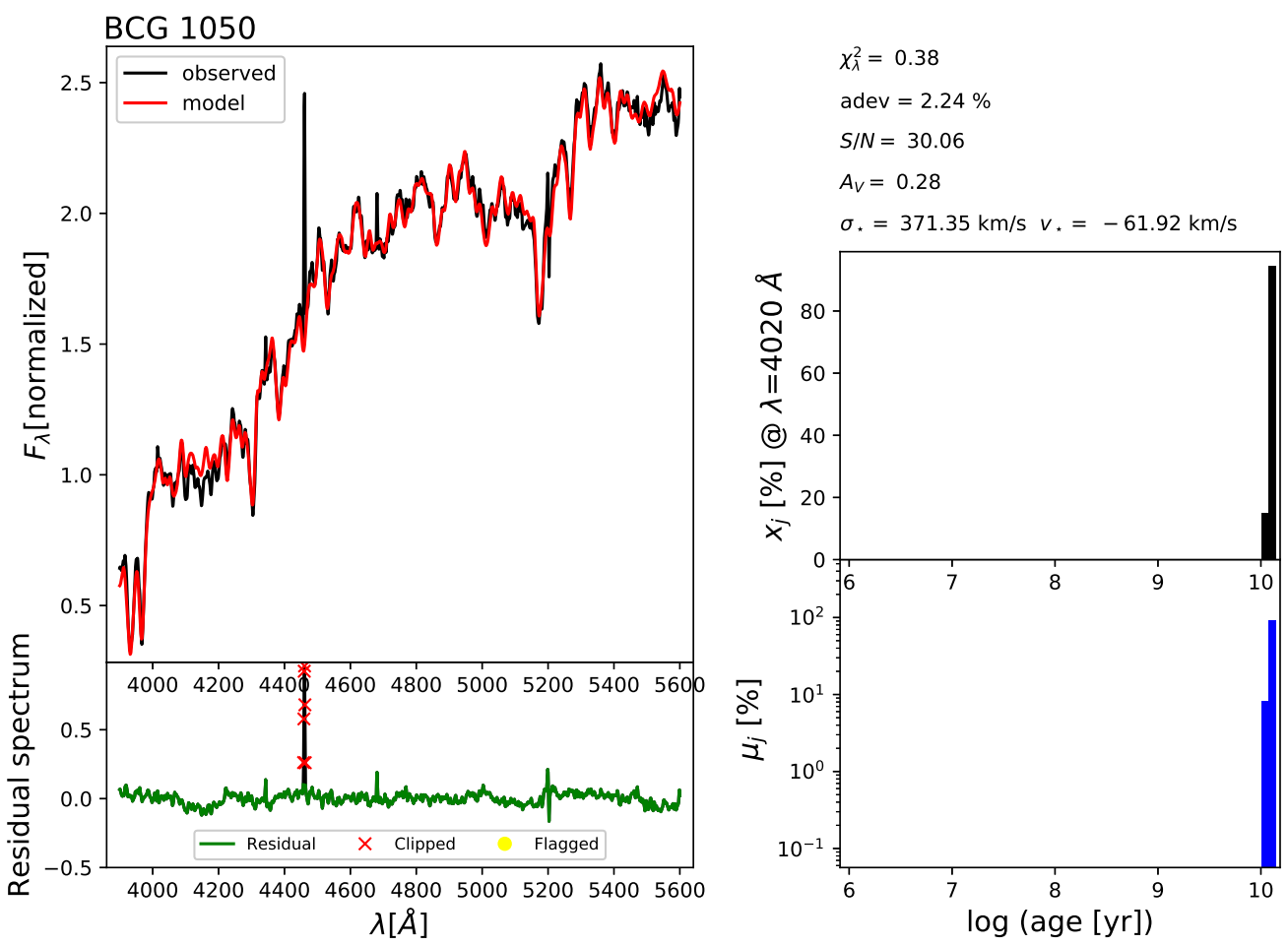

Fig. 3. As Figure 2, but for an old age BCG. The color figure can be viewed online. 

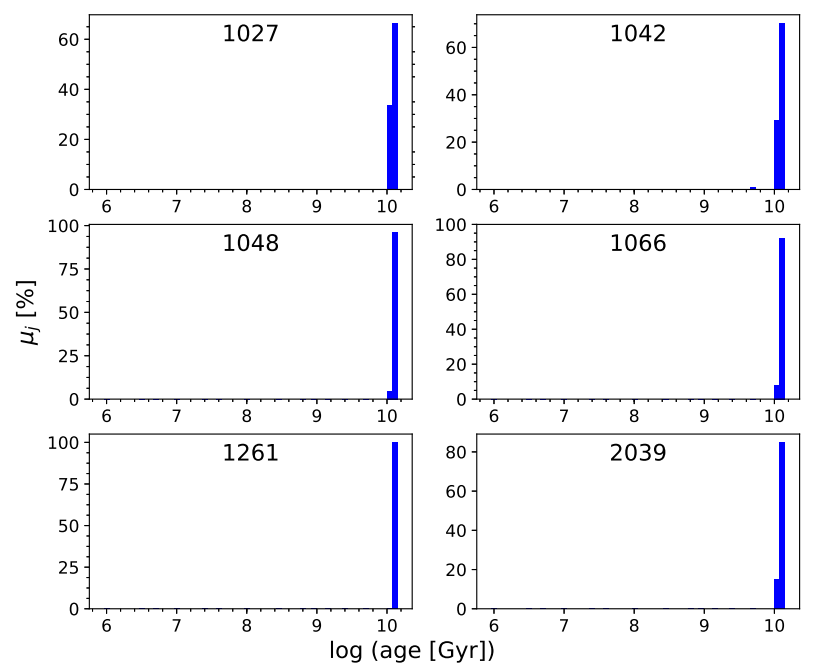

Fig. 4. Star formation histories of BCGs of intermediate central ages. The mass fraction $\mu_{j}$ (obtained by a spectrum fit with STARLIGHT) as a function of logarithm of age. The color figure can be viewed online.

central ages formed $100 \%$ of their stars at $z>2$. The BCG 1042 shows an incipient star formation at $z<2$ (less than $1 \%$ in mass). $100 \%(3 / 3)$ of the BCGs (1050, 2001 and 2086) of old central ages are established with only two stellar populations at $z>2$ (see Figure 5).

\section{DISCUSSION}

In this work, we inferred the SFHs of a sample of BCGs at $z<0.1$ with the objective of determining if these BCGs of intermediate central ages have different accretion histories or if it is only a random effect.

The SFHs found agree with the expectations for elliptical galaxies. These galaxies formed their stars in the early stages of the Universe $(z>2)$ and do not show recent star formation. Most of the SFHs of BCGs were fitted with only two stellar populations of different ages, and no more than four according to the results of the work of Groenewald \& Loubser (2014).

The SFHs of BCGs of intermediate central ages (Figure 4) and old ages (Figure 5) inferred in this work are similar. According to Citro et al. (2016), passive and high mass ETGs $\left(\log \left(\mathrm{M}_{\text {Dyn }}\right)>\right.$ $\left.11.25 M_{\odot}\right)$, ages between approximately $10-13 \mathrm{Gyr}$ and $z$ from 0.06 to 0.08 have SFH similar to those found in our BCGs. McDermid et al. (2015) inferred the SFHs of ETGs from the ATLAS ${ }^{3 \mathrm{D}}$ survey of mass between $11.5<\log \left(\mathrm{M}_{\text {Dyn }}\right)\left[M_{\odot}\right]<12$ and found that $90 \%$ of their stars were formed in the first $3 \mathrm{Gyr}$ $(z \approx 2)$.
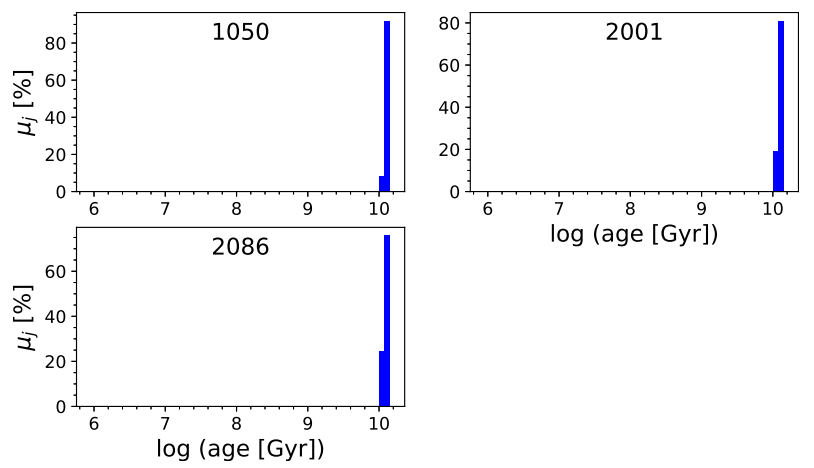

Fig. 5. Star formation histories of BCGs of old central ages. As Figure 4. The color figure can be viewed online.

The results obtained in this work (SFHs showing star formation at $z>2$ ) and Oliva-Altamirano et al. (2015) (high central metallicities and shallow flat metallicity gradients) indicate that the formation and evolution of the BGGs of intermediate ages probably occur through the two-phase scenario. Star formation dominates the mass increase of BCGs at $z>2$ and dry mergers dominate the mass assembly at $z<1$. Jimmy et al. (2013) found that $50 \%$ $(3 / 6)$ of the BCGs of intermediate central ages in our study have experienced recent or ongoing mergers.

Our results are in agreement with semi-analytical models (De Lucia \& Blaizot 2007) and hydrodynamic simulations (Ragone-Figueroa et al. 2018), which propose that star formation in BCGs occurs at $z>2$. Then the BCGs are assembled by dry fusions without significant star formation. These authors disagree on the stellar mass growth rate. Ragone-Figueroa et al. (2018) found a growth rate of 1.3 at $30 \mathrm{kpc}$ and 1.6 at $50 \mathrm{kpc}$, which is significantly lower $(\approx 3)$ than the one proposed by De Lucia \& Blaizot (2007).

The two-phase scenario is supported by several observational studies that have measured the stellar mass growth rate mostly for values less than $z \approx 1$ (e.g. Lidman et al. 2012; Lin et al. 2013; Bellstedt et al. 2016; Zhang et al. 2016). Lidman et al. (2012) showed that BCGs grow by a factor of 1.8 for $z$ between 0.2 and 0.9. Lin et al. (2013) found a similar growth, such that the stellar mass of BCGs increases by a factor of 2.3 from $z \approx 1.4$. Zhang et al. (2016) showed a mass growth of by a factor of $z \approx 2$ from $z \approx 1.2$.

Recently, Edwards et al. (2020) investigated the formation and evolution of local BCGs. They determined stellar populations and dynamics from galaxy core through the outskirts and into the intracluster light. Their results are consistent with the idea that the BCG core and inner regions formed quickly and 
long ago. The outer regions and intracluster light formed more recently, and are continuing to assemble through minor merging.

Based on the above, we conclude that the intermediate central ages of these BCGs do not represent distinct SFHs.

The authors are grateful to Paola Oliva Altamirano and Sarah Brough for comments that led to improvement of this article and data for this research. We thank the STARLIGHT project supported by the Brazilian agencies CNPq, CAPES and FAPESP and by the Frace-Brazilian CAPES/cofecub program.

\section{REFERENCES}

Bai, L., Yee, H. K. C., Yan, R., et al. 2014, ApJ, 789, 134

Bellstedt, S., Lidman, C., Muzzin, A., et al. 2016, MNRAS, 460, 2862

Bernstein, J. P. \& Bhavsar, S. P. 2001, MNRAS, 322, 625

Bruzual, G. \& Charlot, S. 2003, MNRAS, 344, 1000

Cappellari, M., Bacon, R., Bureau, M., et al. 2006, MNRAS, 366, 1126

Cardelli, J. A., Clayton, G. C., \& Mathis, J. S. 1989, ApJ, 345, 245

Cid Fernandes, R., Mateus, A., Sodré, L., Stasińska, G., \& Gomes, J. M. 2005, MNRAS, 358, 363

Citro, A., Pozzetti, L., Moresco, M., \& Cimatti, A. 2016, A\&A, 592, A19

Cowie, L. L. \& Binney, J. 1977, ApJ, 215, 723

De Lucia, G. \& Blaizot, J. 2007, MNRAS, 375, 2

Dressler, A. 1978, ApJ, 223, 765

Dubinski, J. 1998, ApJ, 502, 141

Edwards, L. O. V., Salinas, M., Stanley, S., et al. 2020, MNRAS, 491, 2617

Emsellem, E., Cappellari, M., Krajnović, D., et al. 2007, MNRAS, 379, 401

Fabian, A. C. 1994, ARA\&A, 32, 277

Groenewald, D. N. \& Loubser, S. I. 2014, MNRAS, 444, 808

Hirschmann, M., Naab, T., Davé, R., et al. 2013, MNRAS, 436, 2929

Izzo, C., Kornweibel, N., McKay, D., et al. 2004, Msngr, 117,33

Jimmy, T. K.-V., Brough, S., Gebhardt, K., et al. 2013, ApJ, 778, 171
Katayama, H., Hayashida, K., Takahara, F., \& Fujita, Y. 2003, ApJ, 585, 687

Laine, S., van der Marel, R. P., Lauer, T. R., et al. 2003, AJ, 125,478

Laporte, C. F. P., White, S. D. M., Naab, T., \& Gao, L. 2013, MNRAS, 435, 901

Le Fèvre, O., Saisse, M., Mancini, D., et al. 2003, SPIE, 4841, 1670

Lidman, C., Suherli, J., Muzzin, A., et al. 2012, MNRAS, 427,550

Lin, Y.-T., Brodwin, M., Gonzalez, A. H., et al. 2013, ApJ, 771, 61

Malumuth, E. M., \& Richstone, D. O. 1984, ApJ, 276, 413

McDermid, R. M., Alatalo, K., Blitz, L., et al. 2015, MNRAS, 448, 3484

Merritt, D. 1985, ApJ, 289, 18

Miller, C. J., Nichol, R. C., Reichart, D., et al. 2005, AJ, 130,968

Naab, T., Johansson, P. H., \& Ostriker, J. P. 2009, ApJ, 699, L178

Naab, T., Oser, L., Emsellem, E., et al. 2014, MNRAS, 444,3357

Oemler, A., Jr. 1976, ApJ, 209, 693

Oliva-Altamirano, P., Brough, S., Jimmy, T. K.-V., et al. 2015, MNRAS, 449, 3347

Ostriker, J. P. \& Tremaine, S. D. 1975, ApJ, 202, L113

Peeples, M. S., Werk, J. K., Tumlinson, J., et al. 2014, ApJ, 786, 54

Ragone-Figueroa, C., Granato, G. L., Ferraro, M. E., et al. 2018, MNRAS, 479, 1125

Sandage, A. \& Hardy, E. 1973, ApJ, 183, 743

Schechter, P. L. \& Peebles, P. J. E. 1976, ApJ, 209, 670

Schneider, D. P., Gunn, J. E., \& Hoessel, J. G. 1983, ApJ, 264, 337

Schombert, J. M. 1986, ApJS, 60, 603

Silk, J. 1976, ApJ, 208, 646

Tonini, C., Bernyk, M., Croton, D., Maraston, C., \& Thomas, D. 2012, ApJ, 759, 43

Trager, S. C. \& Somerville, R. S. 2009, MNRAS, 395, 608

Tremaine, S. D. \& Richstone, D. O. 1977, ApJ, 212, 311

Von der Linden, A., Best, P. N., Kauffmann, G., \& White, S. D. M. 2007, MNRAS, 379, 867

White, S. D. M. 1976, MNRAS, 174, 19

York, D. G., Adelman, J., Anderson, J. E., Jr., et al. 2000, AJ, 120, 1579

Zhang, Y., Miller, C., McKay, T., et al. 2016, ApJ, 816, 98

Zhao, D., Aragon-Salamanca, A., \& Conselice, C. J. 2015, VizieR Online Data Catalog, 744

M. L. Talavera and J. Umanzor: Universidad Nacional Autónoma de Honduras, Facultad de Ciencias Espaciales, Departamento de Astronomía y Astrofísica, Edificio K1, Bulevar Suyapa, Tegucigalpa, Honduras, C.A. (martha.talavera, jesus.umanzor@unah.edu.hn). 